\title{
INFLUENCIA DE LOS DILUTORES TRIS Y OVINE FREEZING SOBRE LA INTEGRIDAD DE LA MEMBRANA CITOPLASMÁTICA DURANTE LA CONGELACIÓN DE SEMEN DE OVINOS EN PAJILLAS DE 0.5 ML
}

\author{
Effect of Tris And Ovine Freezing Semen Extenders on Cytoplasmatic \\ Membrane Integrity During Ovine Semen Freezing in 0.5 mL Straws
}

\author{
Próspero Cabrera V. ${ }^{1,2}$ y César Pantoja A. ${ }^{1,3}$
}

\section{Resumen}

Se analizó el efecto de los dilutores Tris-glucosa y Ovine Freezing Buffer (UA 466/ 005238) sobre la motilidad e integridad de la membrana citoplasmática de los espermatozoides durante el proceso de congelación de semen ovino. Se utilizó el semen de cinco carneros (2 Assaf, 2 Canela y 1 Black Belly). El semen fresco fue de buena calidad y los valores de las características seminales estuvieron dentro de los parámetros de la especie. La motilidad individual progresiva (MIP) del semen refrigerado fue $86.0 \pm$ 2.48 y $88.5 \pm 4.8 \%$ y del semen congelado fue de $60.8 \pm 1.9$ y $62.9 \pm 2.4 \%$ con los dilutores Tris y Ovine Freezing, respectivamente; mientras que la proporción de espermatozoides con membrana intacta, evaluada por la prueba de HOST (Hipo Osmotic Swelling Test) fue $77.9 \pm 4.8$ y $78.9 \pm 4.0 \%$ para el semen refrigerado y de $39.9 \pm 3.6$ y $43.2 \pm 2.9 \%$ para el semen congelado, utilizando los dilutores Tris y Ovine Freezing, respectivamente, existiendo diferencias altamente significativas entre dilutores, carneros y fases del proceso de congelación $(p<0.01)$. Se encontró regresiones lineales significativas $(p<0.05)$ entre HOST de semen fresco y HOST post-descongelado de semen diluido, ya sea con Tris o con Ovine Freezing. Se concluye que el uso del dilutor Ovine Freezing tuvo una mejor respuesta en la calidad espermática durante el proceso de congelación; así mismo, la prueba hipoosmótica demostró ser una buena herramienta como indicador de la calidad de semen.

Palabras clave: dilutores, semen congelado, ovinos

\section{Abstract}

The effect of two semen extenders: Glucose Tris and Ovine Freezing Buffer (UA 466/ 005238) on the motility and cytoplasmic membrane integrity of spermatozoa during the freezing process was evaluated. Five rams (2 Assaf, 2 Cinnamon and 1 Black Belly) were used. The fresh semen was of good quality and values of seminal characteristics were within the normal range for this species. The Progressive Individual Motility of the

${ }^{1}$ Departamento de Producción Animal, Universidad Nacional Agraria La Molina, Lima

${ }^{2}$ E-mail: procecavi@lamolina.edu.pe

${ }^{3}$ E-mail: pantoja4444@hotmail.com 
refrigerated semen was $86.0 \pm 2.48$ and $88.5 \pm 4.8 \%$ and for frozen semen was $60.8 \pm 1.9$ and $62.9 \pm 2.4 \%$ for Tris and Ovine Freezing, respectively; while the proportion of spermatozoa with intact membranes, evaluated by HOST (Hipo Osmotic Swelling Test), was 77.9 \pm 4.8 and $78.9 \pm 4.0 \%$ for refrigerated semen and $39.9 \pm 3.6$ and $43.2 \pm 2.9 \%$ for frozen semen using the Tris and Ovine Freezing dilutors, respectively. There were highly significant differences between dilutors, rams and phases of the freezing process $(p<0.01)$. Significant lineal regressions $(\mathrm{p}<0.05)$ were found between HOST values of fresh semen and HOST values of thawed semen, either with Tris or Ovine Freezing. It can be concluded that the Ovine Freezing semen extender showed a better performance during the freezing process; moreover, the hypoosmotic test showed to be a good indicator tool for semen quality.

Key words: extender, frozen semen, ovine

\section{INTRODUCCIÓN}

Los tratados de libre comercio (TLC) con los Estados Unidos y con la Comunidad Económica Europea permitirán satisfacer la demanda de carne de cordero y a precios más competitivos. Esto implica que el avance en genética ovina deberá tener un gran desarrollo en los próximos años, para así mejorar los caracteres hereditarios de interés productivo. En este ámbito, la inseminación artificial es una herramienta que permite realizar un manejo reproductivo eficiente y que juega un rol importante en los programas de mejoramiento genético; y para esto, se deben tener las herramientas de laboratorio necesarias para medir la calidad espermática.

La prueba de permeabilidad de membrana, vía endósmosis o Hipo Osmotic Swelling Test (HOST), está basada en las propiedades físicas y bioquímicas de la membrana plasmática. En la endósmosis positiva se observa como el flagelo del espermatozoide en un medio hipoosmótico (100 mOsm/ $\mathrm{ml}$ ) se torciona helicoidalmente y asciende dentro de la misma membrana celular (Vázquez et al., 1997), debido a un desequilibrio osmótico entre el medio extracelular y el intracelular, situación que el espermatozoide trata de vencer difundiendo agua al compartimiento intracelular y, como consecuencia, la célula aumenta su volumen. Los espermatozoides con membrana funcional muestran un flagelo en curva, mientras que los espermatozoides no viables, mantienen sus colas rectas (Ducci et al., 2002).

En condiciones fisiológicas normales, la fecundación no ocurre si la membrana plasmática del espermatozoide está bioquímicamente inactiva, aún cuando permanezca estructuralmente intacta; por lo tanto, la prueba hipoosmótica es un buen indicador del funcionamiento de la membrana espermática (Tamuli y Watson, 1992); a diferencia de las tinciones vitales, que solo permiten ver la integridad morfológica de la membrana (Brito et al., 2003). En el Perú se han hecho algunas evaluaciones del semen fresco de ovinos en medios hipoosmóticos habiendo obtenido respuestas de $61 \%$ de espermatozoides con membrana intacta que reaccionaron positivo al HOST a los 30 min de incubación (Sandoval et al., 2004).

Durante el proceso de criopreservación se produce una disminución aproximada del $50 \%$ en la viabilidad espermática, debido principalmente al efecto de la temperatura y la presión osmótica, ocurriendo cambios en la organización morfológica de las células, tales como la permeabilidad, composición lipídica de las membranas espermáticas y en el líquido intracelular (Thomas et al., 1998). La hipertonicidad causa desnaturalización proteica, disociación de lipoproteínas e influye en la activación, estabilización o inhibición de enzimas (Meryman, 1966), los cristales de hielo intracelular causan ruptura y 
vacuolización del citoplasma y membranas (Mazur, 1966, 1977), y la ruptura de membranas y, en especial, la de liposomas, está asociada a la liberación de enzimas hidrofílicas liposomales con la consiguiente destrucción química de la célula (Meryman, 1966).

Los dilutores de semen contienen Tris (Hidroximetil Aminometano) o citrato como buffers, glucosa o fructosa como fuente de energía, y penicilina o estreptomicina como agentes antimicrobianos (Gibbons et al., 2004). Además, deben contener agentes protectores para las membranas celulares durante el enfriamiento a $5{ }^{\circ} \mathrm{C}$ (generalmente yema de huevo), y el congelamiento (generalmente glicerol) para evitar lesiones de la membrana durante la congelación (Hafez, 1996). Existen otros dilutores comerciales, como el Laiciphos (IMV, Francia) para diluir semen fresco, Ovine Fresh para semen refrigerado y Ovine Freezing para la congelación de semen.

El objetivo de la presente investigación fue analizar el efecto de dos dilutores (Trisglucosa y Ovine Freezing Buffer) sobre la motilidad e integridad de la membrana citoplasmática de los espermatozoides durante el proceso de congelación.

\section{Materiales y Métodos}

\section{Manejo Animal}

El estudio se realizó en los ambientes del Banco Nacional de Semen de la Universidad Nacional Agraria, ubicado en el distrito de la Molina, Lima. La fase pre-experimental se inició en octubre de 2005 y la fase experimental entre enero y junio de 2006.

Se utilizaron cinco carneros (1 Black Belly, 2 Canela y 2 Assaf), de 3.5 a 4 años de edad, pertenecientes a la Universidad Nacional Agraria La Molina y al Instituto Nacional de Investigación Agraria (INIA). Los carneros se mantuvieron en corrales de $2.8 \mathrm{~m}$ de ancho x $3.0 \mathrm{~m}$ de largo y una altura de 1.2 $\mathrm{m}$, de madera y malla de alambre, provisto de sombras, comedero y bebedero, así como con una adecuada iluminación y ventilación. En cada corral se colocaron 2 animales. La alimentación de los reproductores estuvo basada en forraje verde picado de King grass (Pennisetum purpureum $\mathrm{x}$ Pennisetum typhoides), maíz chala (Zea mays) picado y suplementado con concentrado.

Para la colección de semen en vagina artificial (IMV, Francia), se utilizó una borrega y un brete de monta cuyas dimensiones son de $1.0 \times 0.8 \times 1.2 \mathrm{~m}$ de largo, ancho $\mathrm{y}$ alto, respectivamente, el mismo que cuenta con una fosa por debajo de la superficie de 1.7 x 0.7 x $1.0 \mathrm{~m}$. La temperatura interna de la vagina fue de $38^{\circ} \mathrm{C}$. La frecuencia de colección fue dos veces por semana, obteniéndose un total de ocho eyaculados por carnero.

\section{Evaluación Seminal}

En cada eyaculado se evaluó el volumen, motilidad masal, motilidad individual progresiva, porcentaje de espermatozoides vivos y anormales, concentración espermática y el $\mathrm{pH}$. La evaluación de la integridad de membrana citoplasmática se realizó en el semen fresco, diluido y congelado, mediante la prueba de endósmosis (HOST - Hipo Osmotic Swelling Test) (Correa y Zavos, 1994). Los eyaculados con motilidad baja fueron descartados.

Para la prueba de endósmosis, se mantuvo la pajilla a $37{ }^{\circ} \mathrm{C}$ por $10-15 \mathrm{~s}$ (para el semen refrigerado y congelado), se evaluó la motilidad espermática, se diluyó en una proporción de 1:1 (v/v) de semen en citrato de sodio a1 $2.9 \%$ con $2 \%$ de suero bovino, se centrifugó a 500 rpm por $5 \mathrm{~min}$, y se procedió con la prueba. Para esto, se utilizó una parte de semen por 10 de la solución hipoosmótica a $100 \mathrm{Osmol} / \mathrm{L}$, incubado a $34^{\circ} \mathrm{C}$ por 60 min en baño maría. 


\section{Manipulación del Semen}

Se evaluaron dos tipos de dilutores. En el Tratamiento 1 se utilizó el diluyente Trisglucosa-yema de huevo, propuesto por Evans y Maxwell (1990), y en el Tratamiento 2 se usó el diluyente comercial Ovine Freezing Buffer UA 466/005238, lote $\mathrm{N}^{\circ} \mathrm{S} 98$ (IMV Technologies, France). El número de espermatozoides por pajilla de semen de 0.5 $\mathrm{ml}$ fue de $30 \times 10^{6}$ espermatozoides.

En la congelación del semen se siguió el protocolo descrito por Evans y Maxwell (1990) con algunas variantes basado en los principios de congelación de células referido por Mazur et al. (1972). El descongelamiento se hizo a una temperatura de $38^{\circ} \mathrm{C}$ en termo descongelador, por espacio de $15 \mathrm{~s}$, teniendo especial cuidado en mover la burbuja de aire, secar la pajilla y realizar la evaluación del semen sobre platina temperada.

\section{Análisis Estadístico}

Los valores porcentuales fueron transformados angularmente mediante arco seno. Se empleó el análisis de varianza a través de un diseño de bloques completamente al azar con arreglo factorial 2 × 2 × 5 para evaluar la motilidad e integridad de membrana, siendo los factores a evaluar los dos dilutores (Tris y Ovine Freezing), en dos momentos del procesamiento (refrigerado y congelado), considerando para ello cada carnero (5) como un bloque. El modelo aditivo lineal usado fue el siguiente: $\mathrm{Y}_{\mathrm{ijkl}}=u+\mathrm{B}_{\mathrm{k}}+\mathrm{D}_{\mathrm{i}}+\mathrm{M}_{\mathrm{j}}+\left(\mathrm{D}^{*} \mathrm{M}\right)_{\mathrm{ij}}$ $+\mathrm{e}_{\mathrm{ijk}}+_{\sigma_{\mathrm{ijk}} \mathrm{j}}$, donde:

$\mathrm{y}_{\mathrm{ijkl}} \quad=$ Variable de respuesta de endósmosis positiva del k-ésimo carnero, sujeto al j_ésimo momento de procesamiento de semen con el i-ésimo diluyente.

$\mathrm{u} \quad=$ Media general.

$\mathrm{B}_{\mathrm{k}}=$ Efecto del k-ésimo carnero (Bloques).

$\mathrm{D}_{\mathrm{i}} \quad=$ Efecto del i-ésimo diluyente (Tratamientos).
$\mathrm{M}_{\mathrm{j}} \quad=$ Efecto del $\mathrm{j}$ - ésimo momento en el procesamiento de semen (Refrigerado-Congelado).

$(\mathrm{D} * \mathrm{M})_{\mathrm{ij}}=$ Efecto de la interacción del jésimo momento en el procesamiento de semen sujeto al i-ésimo diluyente.

$\mathrm{e}_{\mathrm{ijk}} \quad=$ Error experimental.

[ $\mathrm{ijkl}=$ Error de muestreo.

Se realizó una prueba de correlación entre los valores de motilidad y HOST del semen fresco, refrigerado y descongelado. Mediante el análisis de regresión lineal se determinó las pruebas que contribuyeron significativamente a explicar la variabilidad observada. Para todos los análisis estadísticos se utilizó el programa SAS (Statistical Analysis System) para un error de 0.05, descrito por Pérez (2001).

\section{Resultados y Discusión}

Las muestras de semen utilizado fueron homogéneas y de buena calidad (Cuadro 1), y se encuentran dentro del rango normal establecido para la especie ovina (Boretto $e t$ al., 2002; Sandoval et al., 2004).

En el Cuadro 2 se muestran los resultados de motilidad individual progresiva del semen fresco y descongelado por tipo de diluyente. La motilidad espermática del semen descongelado fue mejor con el dilutor Ovine Freezing; además, cabe resaltar la nitidez y claridad que brinda este dilutor durante la observación microscópica de la motilidad del semen descongelado. Las diferencias en motilidad entre ambos dilutores estaría muy probablemente influenciado por la proporción de ácido cítrico que contienen los dilutores, ya que este elemento ejerce acción directa en la membrana espermática en la fijación de calcio, que junto con los iones de sodio y potasio, mantienen el equilibrio osmótico favoreciendo la motilidad de los espermatozoides (Borque et al., 1992). 
Cuadro 1. Características espermáticas del semen fresco de cinco carneros reproductores (1 Black Belly, 2 Canela y 2 Assaf) ( $\mathrm{n}=40$ pajillas)

\begin{tabular}{lcc}
\hline Característica & Promedio \pm d.e. & C.V. ${ }^{1}$ \\
\hline Volumen (ml) & $1.7 \pm 0.6$ & 0.33 \\
Motilidad masal (0-5) & $4.3 \pm 0.4$ & 0.10 \\
Espermatozoides vivos (\%) & $82.5 \pm 3.7$ & 0.04 \\
Espermatozoides anormales (\%) & $6.4 \pm 1.4$ & 0.22 \\
Endósmosis positiva (\%) & $81.2 \pm 3.7$ & 0.05 \\
Concentración espermática & 2,735 & 0.24 \\
(millones/ml) & & 0 \\
pH & 7.0 & \\
\hline
\end{tabular}

${ }^{1}$ Coeficiente de variación

Cuadro 2. Motilidad individual progresiva del semen fresco (recién colectado) y descongelado de cinco carneros (Black Belly, Canela y Assaf) almacenado en 20 pajillas de $0.5 \mathrm{ml}$, según tipo de diluyente

\begin{tabular}{ccccc}
\hline \multirow{2}{*}{ Carnero } & \multicolumn{2}{c}{ Semen fresco } & \multicolumn{2}{c}{ Semen descongelado } \\
\cline { 2 - 5 } & $\begin{array}{c}\text { Tris } \\
(\%)\end{array}$ & $\begin{array}{c}\text { Ovine Freezing } \\
(\%)\end{array}$ & $\begin{array}{c}\text { Tris } \\
(\%)\end{array}$ & $\begin{array}{c}\text { Ovine Freezing } \\
(\%)\end{array}$ \\
\hline 1 & $86.6 \pm 1.3$ & $93.2 \pm 1.9$ & $59.1 \pm 2.3^{\mathrm{a}}$ & $61.1 \pm 1.8^{\mathrm{a}}$ \\
2 & $87.8 \pm 1.6$ & $93.2 \pm 1.9$ & $60.5 \pm 1.6^{\mathrm{ab}}$ & $62.6 \pm 3.0^{\mathrm{ab}}$ \\
3 & $82.4 \pm 2.4$ & $81.9 \pm 2.1$ & $60.6 \pm 1.5^{\mathrm{ab}}$ & $63.4 \pm 2.5^{\mathrm{ab}}$ \\
4 & $87.2 \pm 2.1$ & $85.6 \pm 7.3$ & $61.5 \pm 1.6^{\mathrm{b}}$ & $63.4 \pm 2.5^{\mathrm{ab}}$ \\
5 & $86.0 \pm 2.4$ & $88.5 \pm 5.7$ & $62.3 \pm 1.4^{\mathrm{b}}$ & $64.1 \pm 2.2^{\mathrm{b}}$ \\
\hline Total & $86.0 \pm 2.4$ & $88.5 \pm 4.8$ & $60.8 \pm 1.9$ & $62.9 \pm 2.4$ \\
\hline
\end{tabular}

a,b Las letras diferentes dentro de columnas para el semen descongelado indican diferencia significativa $(p<0.05)$

La proporción de espermatozoides con membrana citoplasmática intacta en el semen post-descongelación de los cinco carneros se muestra en el Cuadro 3, observándose una mejor respuesta con el dilutor Ovine Freezing. Como ejemplo, el carnero 1, pese a tener el valor más bajo dentro de cada dilutor, el valor con este dilutor fue adecuado, no así cuando se usó el dilutor Tris. Estos resultados ponen de manifiesto que los efectos de la descongelación están íntimamente relacionados con el proceso de congelación. 
Cuadro 3. Proporción de espermatozoides post-descongelación con membrana citoplasmática intacta en semen de cinco carneros procesado con dos dilutores $(\mathrm{n}=8$ pajillas $)$

\begin{tabular}{ccc}
\hline Carnero & $\begin{array}{c}\text { Tris } \\
(\%)\end{array}$ & $\begin{array}{c}\text { Ovine Freezing } \\
(\%)\end{array}$ \\
\hline 1 & $38.6 \pm 3.4^{\mathrm{ab}}$ & $41.1 \pm 0.8^{\mathrm{a}}$ \\
2 & $39.4 \pm 2.6^{\mathrm{ab}}$ & $42.4 \pm 2.0^{\mathrm{a}}$ \\
3 & $36.9 \pm 1.6^{\mathrm{a}}$ & $43.0 \pm 3.8^{\mathrm{a}}$ \\
4 & $41.0 \pm 1.1^{\mathrm{b}}$ & $43.7 \pm 2.2^{\mathrm{ab}}$ \\
5 & $44.4 \pm 4.2^{\mathrm{c}}$ & $46.2 \pm 2.9^{\mathrm{b}}$ \\
\hline Total & $40.0 \pm 3.6$ & $43.2 \pm 2.9$ \\
\hline
\end{tabular}

${ }^{a, b, c}$ Las letras diferentes dentro de columnas indican diferencia significativa $(p<0.05)$

Cuadro 4. Motilidad individual progresiva del semen de ovino utilizando dos tipos de dilutores durante el proceso de congelación en pajillas de $0.5 \mathrm{ml}$

\begin{tabular}{lcccccc}
\hline \multirow{2}{*}{ Semen } & \multicolumn{3}{c}{ Dilutor Tris } & \multicolumn{3}{c}{ Dilutor Ovine Freezing } \\
\cline { 2 - 7 } & $\mathrm{N}^{\mathrm{o}}$ & $\%$ & $\mathrm{CV}^{1}$ & $\mathrm{~N}^{\mathrm{o}}$ & $\%$ & $\mathrm{CV}$ \\
\hline Refrigerado & 40 & $80.70 \pm 2.75$ & 0.03 & 40 & $82.55 \pm 2.74$ & 0.03 \\
Congelado & 40 & $60.77 \pm 1.89$ & 0.03 & 40 & $62.93 \pm 2.41$ & 0.04 \\
\hline${ }^{1}$ Coeficiente de variación & & & & &
\end{tabular}

Cuadro 5. Endósmosis de semen ovino en diferentes momentos del proceso de congelación utilizando dos tipos de dilutores $(n=40$ pajillas por observación)

\begin{tabular}{lcccccc}
\hline \multirow{2}{*}{ Semen } & \multicolumn{2}{c}{ Sin diluir } & \multicolumn{2}{c}{ Dilutor Tris } & \multicolumn{2}{c}{ Dilutor Ovine Freezing } \\
\cline { 2 - 7 } & $\%$ & $\mathrm{CV}^{1}$ & $\%$ & $\mathrm{CV}$ & $\%$ & $\mathrm{CV}$ \\
\hline Fresco & $81.2 \pm 3.7$ & 0.05 & & & & \\
Refrigerado & & & $77.9 \pm 4.8$ & 0.06 & $78.9 \pm 4.0$ & 0.05 \\
Congelado & & & $39.9 \pm 3.6$ & 0.09 & $43.2 \pm 2.9$ & 0.07 \\
\hline
\end{tabular}

${ }^{1}$ Coeficiente de variación 
En el Cuadro 4, se aprecia el porcentaje de espermatozoides con movimiento progresivo en los diferentes periodos de procesamiento del semen. Se encontró diferencia estadística altamente significativa $(\mathrm{p}<0.01)$ entre dilutores, entre carneros y entre momentos de procesamiento, pero no en la interacción momento - dilutor.

En el Cuadro 5 se muestra el porcentaje de espermatozoides que reaccionaron en forma positiva a la prueba de endósmosis durante el procesamiento de congelación del semen. La tasa del endósmosis en semen descongelado se redujo en $50 \%$ respecto al semen fresco, porcentaje similar a otros reportes (Thomas et al., 1998), quienes afirman que durante el proceso de criopreservación se produce esta disminución en la viabilidad espermática, debido principalmente al efecto de la temperatura y a la presión osmótica, ocurriendo cambios en la organización morfológica de las células, tales como la permeabilidad, composición lipídica de las membranas espermáticas y en el liquido intracelular.

Se encontraron regresiones lineales significativas entre los valores de HOST en semen fresco con el valor de Host en semen refrigerado utilizando el dilutor Tris $(p<0.05)$ y Ovine Freezing $(\mathrm{p}<0.01)$, así como entre la motilidad del semen refrigerado con el valor de HOST del semen congelado utilizando en ambos casos el dilutor Tris ( $\mathrm{p}<0.05$ ). Las demás regresiones no fueron significativas. Esto es indicativo que la motilidad seminal, dentro de rangos adecuados, no es un buen indicador de la calidad de semen. Por otro lado, el volumen de eyaculado no tiene correlaciones significativas con otras características espermáticas; la motilidad masal tiene una baja correlación con la concentración $(+0.216)$ y muy baja con el porcentaje de espermatozoides anormales $(+0.1)$ y es negativa con el resto de las características; la concentración espermática mantiene una correlación negativa con todas las características, salvo la motilidad que es baja; el porcentaje de espermatozoides vivos no correlaciona con ninguna de las características evaluadas, salvo con el porcentaje de endósmosis en semen fresco que es muy alta $(+0.92)$ $(p<0.01)$; y el porcentaje de espermatozoides anormales presenta una baja correlación con motilidad, siendo negativa con el resto de características espermáticas.

La correlación negativa entre los valores presentados por la motilidad masal microscópica y el Host en semen fresco (-0.22, $\mathrm{p}=0.1631>0.05$ ), indicaría que una variación en el movimiento masal no estaría asociada a un incremento o una disminución en la proporción de espermatozoides con membrana citoplasmática intacta (viables). Investigaciones de referencia sugieren la existencia de una subpoblación de espermatozoides que aún con su membrana dañada presentaría movilidad. Este grupo podría estar contribuyendo a la presencia e intensidad de movimiento en masa post-descongelamiento y reaccionar negativamente al Host (Boretto et al., 2002).

\section{ConClusiones}

- La motilidad del semen luego de la descongelación, muestra una diferencia altamente significativa $(\mathrm{p}<0.01)$ a favor del dilutor Ovine Freezing, así como entre carneros y en las fases del proceso de congelación.

- La prueba hipoosmótica (HOST), aplicada para determinar la integridad de membrana citoplasmática, permitió evidenciar diferencias significativas $(p<0.01)$ entre dilutores, entre carneros $y$ en las fases del proceso de congelación.

\section{Literatura Citada}

1. Boretto JM, Gibbons AE, Bunge MM, Cueto MI, Bidinodt F. 2002. Calidad seminal post descongelamiento en relación con la eficiencia reproductiva de la inseminación artificial laparoscópica en 
ovinos. Rev Med Vet, Argentina 83(4): 185-188.

2. Borque M, Saguez A. 1992. Variaciones estacionales de los niveles de fructosa, ácido cítrico y proteínas totales en eyaculados de moruecos de raza Manchega. Invest Agr Prod Sanid Anim 7(3): 235-240.

3. Brito LFC, Barth AD, BilodeauGoeseels S, Panich PL, Kastelic JP. 2003. Comparison of methods to evaluate the plasmalemma of bovine sperm and their relationship with in vitro fertilization rate. Theriogenology 60: 1539-1551.

4. Correa JR, Zavos PM. 1994. The hipoosmotic swelling test: Its employment as an assay to evaluate the functional integrity of the frozen-thawed sperm characteristics assessed via the routine semen analysis, sperm functional test and fertility of bulls in an artificial insemination program. Theriogenology 48: 721-731.

5. Correa J, Pace M, Zavos P. 1997. Relationship among frozen-thawed sperm characteristics assessed via the routine semen analysis, sperm functional test and fertility of bulls in an artificial insemination program. Theriogenology 48: 721-731.

6. Ducci M, Gazzano A, Villani C, Cela V, Artini PG, Martelli F, Genazzani AR. 2002. Membrane integrity evaluation in rabbit spermatozoa. Eur J Obst Gynecol Reprod Biol [Internet], [10 abril 2007]. Disponible en: http://www.ncbi.nlm.nih. gov/entrezlquery.fcgi

7. Evans G, Maxwell WMC. 1990. Salamon's artificial insemination of sheep and goats». London: Butterworth. $192 \mathrm{p}$.

8. Gibbons A, Cueto M, Wolf M, Arrigo J, Garcia J. 2004. Obtención, procesamiento y conservación del semen ovino. Comunicación Técnica PA 200. INTA. EEA Bariloche. 23 p.

9. Hafez ESE. 1996. Reproducción e inseminación artificial en animales. $6^{\mathrm{a}}$ ed.
México: Interamericana McGraw-Hill. $694 \mathrm{p}$.

10. Mazur P. 1966. Physical and chemical basis of injury in single-celled microorganisms subjected to freezing and thawing. In: Meryman HT. Cryobiology. New York: Academic press. p 213-315.

11. Mazur P. 1977. The role of intracellular freezing in the death of cells cooled at supraoptimal rates. Cryobiology 14: 251272.

12. Mazur P, Leibo SP, Chu EHY. 1972. A two factor hypothesis of freezing injury. Evidence from Chinese hamster tissue culture cells. Expl Cell Res 71: 345355.

13. Meryman HT. 1966. Review of biological freezing. In: Meryman HT. Cryobiology. New York: Academic press. p 1-114.

14. Pérez LC. 2001. El sistema estadístico SAS. España: Ed. Prentice Hall. 773 p.

15. Sandoval MR, Santiani AA, Ruiz GL, Leyva VV, Coronado LS, Delgado AC. 2007. Criopreservación de semen ovino empleando tres dilutores y cuatro combinaciones de agentes crioprotectores permeantes y no permeantes. Rev Inv Vet, Perú 18: 107-114.

16. Tamuli MK, Watson PF. 1992. Effects of temperature of incubation on the development of resistance to cold stress in boar spermatozoa incubated for up 24 hours. Proc $12^{\text {th }}$ ICAR Congress. The Hague. p 1484-1486.

17. Thomas CA, Garner DL, Dejarnette JM, Marshall CE. 1998. Effect of cryopreservation on bovine sperm organelle function and viability as determined by flow cytometry. Biol Reprod 58: 786-793.

18. Vásquez JM, Martínez EA, Roca J, Blanco O, Lucas X, Matas C. 1997. Hypoosmotic swelling of boar spermatozoa compared to other methods for analyzing the sperm membrane. Theriogenology 47: 913-922. 